\title{
General Deviants: An Analysis of Perturbations in Compressed Sensing
}

\author{
Matthew A. Herman and Thomas Strohmer
}

\begin{abstract}
We analyze the Basis Pursuit recovery of signals with general perturbations. Previous studies have only considered partially perturbed observations $\boldsymbol{A} \boldsymbol{x}+\boldsymbol{e}$. Here, $\boldsymbol{x}$ is a signal which we wish to recover, $\boldsymbol{A}$ is a full-rank matrix with more columns than rows, and $\boldsymbol{e}$ is simple additive noise. Our model also incorporates perturbations $\boldsymbol{E}$ to the matrix $\boldsymbol{A}$ which result in multiplicative noise. This completely perturbed framework extends the prior work of Candès, Romberg and Tao on stable signal recovery from incomplete and inaccurate measurements. Our results show that, under suitable conditions, the stability of the recovered signal is limited by the noise level in the observation. Moreover, this accuracy is within a constant multiple of the best-case reconstruction using the technique of least squares. In the absence of additive noise numerical simulations essentially confirm that this error is a linear function of the relative perturbation.
\end{abstract}

\section{INTRODUCTION}

Employing the techniques of compressed sensing (CS) to recover signals with a sparse representation has enjoyed a great deal of attention over the last 5-10 years. The initial studies considered an ideal unperturbed scenario:

$$
\boldsymbol{b}=\boldsymbol{A x} .
$$

Here $\boldsymbol{b} \in \mathbb{C}^{m}$ is the observation vector, $\boldsymbol{A} \in \mathbb{C}^{m \times n}$ is a full-rank measurement matrix or system model (with $m \leq n$ ), and $\boldsymbol{x} \in \mathbb{C}^{n}$ is the signal of interest which has a sparse, or almost sparse, representation under some fixed basis. More recently researchers have included an additive noise term $e$ into the received signal [1]-[4] creating a partially perturbed model:

$$
\hat{b}=A x+e
$$

This type of noise typically models simple errors which are uncorrelated with $\boldsymbol{x}$.

As far as we can tell, practically no research has been done yet on perturbations $\boldsymbol{E}$ to the matrix $\boldsymbol{A}$, 1, 2 Our completely perturbed model extends (2) by incorporating a perturbed sensing matrix in the form of

$$
\hat{\boldsymbol{A}}=\boldsymbol{A}+\boldsymbol{E} .
$$

It is important to consider this kind of noise since it can account for precision errors when applications call for physically implementing the measurement matrix $\boldsymbol{A}$ in a sensor. In other CS scenarios, such as when $\boldsymbol{A}$ represents a system model, $\boldsymbol{E}$ can absorb errors in assumptions made about the transmission channel. This can be realized in radar [7], remote sensing [8], telecommunications, source separation [5], [6], and countless other problems. Further, $\boldsymbol{E}$ can also model the distortions that result when discretizing the domain of analog signals and systems; examples include jitter error and choosing too coarse of a sampling period.

The authors are with the Department of Mathematics, University of California, Davis, CA 95616-8633, USA (e-mail: \{mattyh, strohmer\}emath.ucdavis.edu).

This work was partially supported by NSF Grant No. DMS-0811169 and NSF VIGRE Grant No. DMS-0636297.

${ }^{1} \mathrm{~A}$ related problem is considered in [5] for greedy algorithms rather than $\ell_{1}$-minimization, and in a multichannel rather than a single channel setting; it mentions using different matrices on the encoding and decoding sides, but its analysis is not from an error or perturbation point of view.

${ }^{2}$ At the time of revising this manuscript we became aware of an earlier study [6] which discusses the error resulting from estimating the mixing matrix in source separation problems. However, it only covers strictly sparse signals, and its analysis is not as in depth as presented in this manuscript. 
In general, these perturbations can be characterized as multiplicative noise, and are more difficult to analyze than simple additive noise since they are correlated with the signal of interest. To see this, simply substitute $\boldsymbol{A}=\hat{\boldsymbol{A}}-\boldsymbol{E}$ in (2) 3 there will be an extra noise term $\boldsymbol{E x}$.

The rest of this section establishes certain assumptions and notation necessary for our analysis. Section $\amalg$ first gives a brief review of previous work on the partially perturbed scenario in CS, and then presents our main theoretical and numerical results on the completely perturbed scenario. Section and Section IV compares the CS solution with classical least squares. Concluding remarks are given in Section $\nabla$ and a brief discussion on different kinds of perturbation $\boldsymbol{E}$ which we often encounter can be found in the Appendix.

\section{A. Assumptions and Notation}

Throughout this paper we represent vectors and matrices with boldface type. Without loss of generality, assume that the original data $\boldsymbol{x}$ is a $K$-sparse vector for some fixed $K$, or that it is compressible. Vectors which are $K$ sparse contain no more than $K$ nonzero elements, and compressible vectors are ones whose ordered coefficients decay according to a power law (i.e., $|\boldsymbol{x}|_{(k)} \leq C_{p} k^{-p}$, where $|\boldsymbol{x}|_{(k)}$ is the $k$ th largest element of $\boldsymbol{x}, p \geq 1$, and $C_{p}$ is a constant which depends only on $p$ ). Let vector $\boldsymbol{x}_{K} \in \mathbb{C}^{n}$ be the best $K$-term approximation to $\boldsymbol{x}$, i.e., it contains the $K$ largest coefficients of $\boldsymbol{x}$ with the rest set to zero. We occasionally refer to this vector as the "head" of $\boldsymbol{x}$. Note that if $\boldsymbol{x}$ is $K$-sparse, then $\boldsymbol{x}=\boldsymbol{x}_{K}$. With a slight abuse of notation denote $\boldsymbol{x}_{K^{c}}=\boldsymbol{x}-\boldsymbol{x}_{K}$ as the "tail" of $\boldsymbol{x}$.

The symbols $\sigma_{\max }(\boldsymbol{Y}), \sigma_{\min }(\boldsymbol{Y})$, and $\|\boldsymbol{Y}\|_{2}$ respectively denote the usual maximum, minimum nonzero singular values, and spectral norm of a matrix $\boldsymbol{Y}$. Our analysis will require examination of submatrices consisting of an arbitrary collection of $K$ columns. We use the superscript $(K)$ to represent extremal values of the above spectral measures. For instance, $\sigma_{\max }^{(K)}(\boldsymbol{Y})$ denotes the largest singular value taken over all $K$-column submatrices of $\boldsymbol{Y}$. Similar definitions apply to $\|\boldsymbol{Y}\|_{2}^{(K)}$ and $\operatorname{rank}^{(K)}(\boldsymbol{Y})$, while $\sigma_{\min }^{(K)}(\boldsymbol{Y})$ is the smallest nonzero singular value over all $K$-column submatrices of $\boldsymbol{Y}$. With these, the perturbations $\boldsymbol{E}$ and $\boldsymbol{e}$ can be quantified with the following relative bounds

$$
\frac{\|\boldsymbol{E}\|_{2}}{\|\boldsymbol{A}\|_{2}} \leq \varepsilon_{\boldsymbol{A}}, \quad \frac{\|\boldsymbol{E}\|_{2}^{(K)}}{\|\boldsymbol{A}\|_{2}^{(K)}} \leq \varepsilon_{\boldsymbol{A}}^{(K)}, \quad \frac{\|\boldsymbol{e}\|_{2}}{\|\boldsymbol{b}\|_{2}} \leq \varepsilon_{\boldsymbol{b}},
$$

where $\|\boldsymbol{A}\|_{2},\|\boldsymbol{A}\|_{2}^{(K)},\|\boldsymbol{b}\|_{2} \neq 0$. In real-world applications we often do not know the exact nature of $\boldsymbol{E}$ and $\boldsymbol{e}$ and instead are forced to estimate their relative upper bounds. This is the point of view taken throughout most of this treatise. In this study we are only interested in the case where $\varepsilon_{\boldsymbol{A}}, \varepsilon_{\boldsymbol{A}}^{(K)}, \varepsilon_{\boldsymbol{b}}<1$.

\section{CS $\ell_{1}$ Perturbation Analysis}

\section{A. Previous Work}

In the partially perturbed scenario (i.e., $\boldsymbol{E}=\mathbf{0}$ ) we are concerned with solving the Basis Pursuit (BP) problem [9]:

$$
\boldsymbol{z}^{\star}=\underset{\hat{z}}{\operatorname{argmin}}\|\hat{\boldsymbol{z}}\|_{1} \quad \text { s.t. }\|\boldsymbol{A} \hat{\boldsymbol{z}}-\hat{\boldsymbol{b}}\|_{2} \leq \varepsilon^{\prime}
$$

for some $\varepsilon^{\prime} \geq 0.4$

The restricted isometry property (RIP) [10] for any matrix $\boldsymbol{A} \in \mathbb{C}^{m \times n}$ defines, for each integer $K=1,2, \ldots$, the restricted isometry constant (RIC) $\delta_{K}$, which is the smallest nonnegative number such that

$$
\left(1-\delta_{K}\right)\|\boldsymbol{x}\|_{2}^{2} \leq\|\boldsymbol{A} \boldsymbol{x}\|_{2}^{2} \leq\left(1+\delta_{K}\right)\|\boldsymbol{x}\|_{2}^{2}
$$

holds for any $K$-sparse vector $\boldsymbol{x}$. In the context of the RIC, we observe that $\|\boldsymbol{A}\|_{2}^{(K)}=\sigma_{\max }^{(K)}(\boldsymbol{A}) \leq \sqrt{1+\delta_{K}}$, and $\sigma_{\min }^{(K)}(\boldsymbol{A}) \geq \sqrt{1-\delta_{K}}$

\footnotetext{
${ }^{3}$ It essentially makes no difference whether we account for the perturbation $\boldsymbol{E}$ on the "encoding side" (2), or on the "decoding side" (7). The model used here was chosen so as to agree with the conventions of classical perturbation theory which we use in Section IV]

${ }^{4}$ Throughout this paper absolute errors are denoted with a prime. In contrast, relative perturbations, such as in (3), are not primed.
} 
Assuming $\delta_{2 K}<\sqrt{2}-1$ and $\|\boldsymbol{e}\|_{2} \leq \varepsilon^{\prime}$, Candès has shown ([1], Thm. 1.2) that the solution to (4) obeys

$$
\left\|\boldsymbol{z}^{\star}-\boldsymbol{x}\right\|_{2} \leq C_{0} K^{-1 / 2}\left\|\boldsymbol{x}-\boldsymbol{x}_{K}\right\|_{1}+C_{1} \varepsilon^{\prime}
$$

for some constants $C_{0}, C_{1} \geq 0$ which are reasonably well-behaved and can be calculated explicitly.

\section{B. Incorporating nontrivial perturbation $\boldsymbol{E}$}

Now assume the completely perturbed situation with $\boldsymbol{E}, \boldsymbol{e} \neq \mathbf{0}$. In this case the BP problem of (4) can be generalized to include a different decoding matrix $\hat{A}$ :

$$
\boldsymbol{z}^{\star}=\underset{\hat{\boldsymbol{z}}}{\operatorname{argmin}}\|\hat{\boldsymbol{z}}\|_{1} \text { s.t. }\|\hat{\boldsymbol{A}} \hat{\boldsymbol{z}}-\hat{\boldsymbol{b}}\|_{2} \leq \boldsymbol{\varepsilon}_{\boldsymbol{A}, K, \boldsymbol{b}}^{\prime}
$$

for some $\varepsilon_{\boldsymbol{A}, K, \boldsymbol{b}}^{\prime} \geq 0$. The following two theorems summarize our results.

Theorem 1 (RIP for $\hat{A})$. Fix $K=1,2, \ldots$ Given the RIC $\delta_{K}$ associated with matrix $\boldsymbol{A}$ in (5) and the relative perturbation $\varepsilon_{\boldsymbol{A}}^{(K)}$ associated with (possibly unknown) matrix $\boldsymbol{E}$ in (3), fix the constant

$$
\hat{\delta}_{K, \max }:=\left(1+\delta_{K}\right)\left(1+\varepsilon_{\boldsymbol{A}}^{(K)}\right)^{2}-1 .
$$

Then the RIC $\hat{\delta}_{K}$ for matrix $\hat{\boldsymbol{A}}=\boldsymbol{A}+\boldsymbol{E}$ is the smallest nonnegative number such that

$$
\left(1-\hat{\delta}_{K}\right)\|\boldsymbol{x}\|_{2}^{2} \leq\|\hat{\boldsymbol{A}} \boldsymbol{x}\|_{2}^{2} \leq\left(1+\hat{\delta}_{K}\right)\|\boldsymbol{x}\|_{2}^{2}
$$

holds for any $K$-sparse vector $\boldsymbol{x}$ where $\hat{\delta}_{K} \leq \hat{\delta}_{K, \max }$.

Remark 1. Properly interpreting Theorem 2 is important. It is assumed that the only information known about matrix $\boldsymbol{E}$ is its worst-case relative perturbation $\varepsilon_{\boldsymbol{A}}^{(K)}$, and therefore the bound of $\hat{\delta}_{K, \max }$ in (8) represents a worstcase deviation of $\hat{\delta}_{K}$. Notice for a given $\varepsilon_{\boldsymbol{A}}^{(K)}$ that there are infinitely many $\boldsymbol{E}$ which satisfy it. In fact, it is possible to construct nonzero perturbations which result in $\hat{\delta}_{K}=\delta_{K}$ ! For example, suppose $\hat{\boldsymbol{A}}=\boldsymbol{A U}$ for some unitary matrix $\boldsymbol{U} \neq \boldsymbol{I}$ where $\boldsymbol{I}$ is the identity matrix. Clearly here $\boldsymbol{E}=\boldsymbol{A}(\boldsymbol{U}-\boldsymbol{I}) \neq \mathbf{0}$ and yet since $\boldsymbol{U}$ is unitary we have $\hat{\delta}_{K}=\delta_{K}$. In this case using $\varepsilon_{\boldsymbol{A}}^{(K)}$ to calculate $\hat{\delta}_{K, \text { max }}$ could be a gross upper bound for $\hat{\delta}_{K}$. If more information on $\boldsymbol{E}$ is known $\sqrt[5]{5}$ then much tighter bounds on $\hat{\delta}_{K}$ can be determined.

Remark 2. The flavor of the RIP is defined with respect to the square of the operator norm. That is, $\left(1-\delta_{K}\right)$ and $\left(1+\delta_{K}\right)$ are measures of the square of the minimum and maximum singular values of $K$-column submatrices of $\boldsymbol{A}$, and similarly for $\hat{\boldsymbol{A}}$. In keeping with the convention of classical perturbation theory however, we defined $\varepsilon_{A}^{(K)}$ in (3) just in terms of the operator norm (not its square). Therefore, the quadratic dependence of $\hat{\delta}_{K, \text { max }}$ on $\varepsilon_{\boldsymbol{A}}^{(K)}$ in (8) makes sense. Moreover, in discussing the spectrum of $K$-column submatrices of $\hat{A}$, we see that it is really a linear function of $\varepsilon_{\boldsymbol{A}}^{(K)}$.

Before introducing the next theorem let us define the following constants due to matrix $\boldsymbol{A}$

$$
\kappa_{\boldsymbol{A}}^{(K)}:=\frac{\sqrt{1+\delta_{K}}}{\sqrt{1-\delta_{K}}}, \quad \alpha_{\boldsymbol{A}}:=\frac{\|\boldsymbol{A}\|_{2}}{\sqrt{1-\delta_{K}}} .
$$

The first quantity bounds the ratio of the extremal singular values of all $K$-column submatrices of $\boldsymbol{A}$

$$
\frac{\sigma_{\max }^{(K)}(\boldsymbol{A})}{\sigma_{\min }^{(K)}(\boldsymbol{A})} \leq \kappa_{\boldsymbol{A}}^{(K)} \text {. }
$$

Actually, for very small $\delta_{K}$ we have $\kappa_{\boldsymbol{A}}^{(K)} \approx 1$, which implies that every $K$-column submatrix forms an approximately orthonormal set.

Also introduce the ratios

$$
r_{K}:=\frac{\left\|\boldsymbol{x}_{K^{c}}\right\|_{2}}{\left\|\boldsymbol{x}_{K}\right\|_{2}}, \quad s_{K}:=\frac{\left\|\boldsymbol{x}_{K^{c}}\right\|_{1}}{\left\|\boldsymbol{x}_{K}\right\|_{2}}
$$

\footnotetext{
${ }^{5}$ See the appendix for more discussion on the different forms of perturbation $\boldsymbol{E}$ which we are likely to encounter.
} 
which quantify the weight of a signal's tail relative to its head. When $\boldsymbol{x}$ is $K$-sparse we have $\boldsymbol{x}_{K^{c}}=\mathbf{0}$, and so $r_{K}=s_{K}=0$. If $\boldsymbol{x}$ is compressible, then these values are a function of the power $p$ (i.e., the rate at which the coefficients decay), and the cardinality $K$ of the group of its largest entries. For reasonable values of $p$ and $K$, we expect that $r_{K}, s_{K} \ll 1$.

Theorem 2 (Stability from completely perturbed observation). Fix the relative perturbations $\varepsilon_{\boldsymbol{A}}, \varepsilon_{\boldsymbol{A}}^{(K)}$, $\varepsilon_{\boldsymbol{A}}^{(2 K)}$ and $\varepsilon_{\boldsymbol{b}}$ in (3). Assume the RIC for matrix $\boldsymbol{A}$ satisfies

$$
\delta_{2 K}<\frac{\sqrt{2}}{\left(1+\varepsilon_{\boldsymbol{A}}^{(2 K)}\right)^{2}}-1,
$$

and that general signal $\boldsymbol{x}$ satisfies

$$
r_{K}+\frac{s_{K}}{\sqrt{K}}<\frac{1}{\kappa_{\boldsymbol{A}}^{(K)}}
$$

Set the total noise parameter

$$
\varepsilon_{\boldsymbol{A}, K, \boldsymbol{b}}^{\prime}:=\left(\frac{\varepsilon_{\boldsymbol{A}}^{(K)} \kappa_{\boldsymbol{A}}^{(K)}+\varepsilon_{\boldsymbol{A}} \alpha_{\boldsymbol{A}} r_{K}}{1-\kappa_{\boldsymbol{A}}^{(K)}\left(r_{K}+s_{K} / \sqrt{K}\right)}+\varepsilon_{\boldsymbol{b}}\right)\|\boldsymbol{b}\|_{2} .
$$

Then the solution of the BP problem (7) obeys

$$
\left\|\boldsymbol{z}^{\star}-\boldsymbol{x}\right\|_{2} \leq \frac{C_{0}}{\sqrt{K}}\left\|\boldsymbol{x}-\boldsymbol{x}_{K}\right\|_{1}+C_{1} \varepsilon_{\boldsymbol{A}, K, \boldsymbol{b}}^{\prime}
$$

where

$$
\begin{gathered}
C_{0}=\frac{2\left(1+(\sqrt{2}-1)\left[\left(1+\delta_{2 K}\right)\left(1+\varepsilon_{\boldsymbol{A}}^{(2 K)}\right)^{2}-1\right]\right)}{1-(\sqrt{2}+1)\left[\left(1+\delta_{2 K}\right)\left(1+\varepsilon_{\boldsymbol{A}}^{(2 K)}\right)^{2}-1\right]}, \\
C_{1}=\frac{4 \sqrt{1+\delta_{2 K}}\left(1+\varepsilon_{\boldsymbol{A}}^{(2 K)}\right)}{1-(\sqrt{2}+1)\left[\left(1+\delta_{2 K}\right)\left(1+\varepsilon_{\boldsymbol{A}}^{(2 K)}\right)^{2}-1\right]} .
\end{gathered}
$$

Remark 3. Theorem 2 generalizes Candès' results in [1]. Indeed, if matrix $\boldsymbol{A}$ is unperturbed, then $\boldsymbol{E}=\mathbf{0}$ and $\varepsilon_{\boldsymbol{A}}=\varepsilon_{\boldsymbol{A}}^{(K)}=0$. It follows that $\hat{\delta}_{K}=\delta_{K}$ in $(8)$, and the RIPs for $\boldsymbol{A}$ and $\hat{\boldsymbol{A}}$ coincide. Moreover, assumption (12) in Theorem 2 reduces to $\delta_{K}<\sqrt{2}-1$, and the total perturbation (see (23)) collapses to $\|\boldsymbol{e}\|_{2} \leq \varepsilon_{\boldsymbol{b}}^{\prime}:=\varepsilon_{\boldsymbol{b}}\|\boldsymbol{b}\|_{2}$ (so that assumption (13) is no longer necessary); both of these are identical to Candès' assumptions in (6). Finally, the constants $C_{0}, C_{1}$ in (16) and (17) reduce to the same as outlined in the proof of [1].

The assumption in (13) demands more discussion. Observe that the left-hand side (LHS) is solely a function of the signal $\boldsymbol{x}$, while the right-hand side (RHS) is just a function of the matrix $\boldsymbol{A}$. For reasonably compressible signals, it is often the case that the LHS is on the order of $10^{-2}$ or $10^{-3}$. At the same time, the RHS is always of order $10^{0}$ due to assumption (12). Therefore, there should be a sufficient gap to ensure that assumption (13) holds. Clearly this condition is automatically satisfied whenever $\boldsymbol{x}$ is strictly $K$-sparse.

In fact, more can be said about Theorem 2 for the case of a $K$-sparse input. Notice then that the terms related to $x_{K^{c}}$ in (14) and (15) disappear, and the accuracy of the solution becomes

$$
\left\|\boldsymbol{z}^{\star}-\boldsymbol{x}\right\|_{2} \leq C_{1}\left(\kappa_{\boldsymbol{A}}^{(K)} \varepsilon_{\boldsymbol{A}}^{(K)}+\varepsilon_{\boldsymbol{b}}\right)\|\boldsymbol{b}\|_{2} .
$$

This form of the stability of the BP solution is helpful since it highlights the effect of the perturbation $\boldsymbol{E}$ on the $K$ most important elements of $\boldsymbol{x}$, as well as the influence of the additive noise $\boldsymbol{e}$. Clearly in the absence of any perturbation, a $K$-sparse signal can be perfectly recovered by BP.

It is also interesting to examine the spectral effects due to the first assumption of Theorem 2. Namely, we want to be assured that the maximum rank of submatrices of $\boldsymbol{A}$ is unaltered by the perturbation $\boldsymbol{E}$.

${ }^{6}$ Note for $\delta_{2 K} \geq 0$, (12) requires that $\varepsilon_{\boldsymbol{A}}^{(2 K)}<\sqrt[4]{2}-1$. 
Lemma 1. Assume condition (12) of Theorem 2 holds. Then for any $k \leq 2 \mathrm{~K}$

$$
\sigma_{\max }^{(k)}(\boldsymbol{E})<\sigma_{\min }^{(k)}(\boldsymbol{A}),
$$

and therefore

$$
\operatorname{rank}^{(k)}(\hat{\boldsymbol{A}})=\operatorname{rank}^{(k)}(\boldsymbol{A}) .
$$

We apply this fact in the least squares analysis of Section IV

The utility of Theorems 1 and 2 can be understood with two simple numerical examples. Suppose that matrix $\boldsymbol{A}$ in (2) represents a system that a signal passes through which in reality has an RIC of $\delta_{2 K}=0.100$. Assume however, that when modeling this system we introduce a worst-case relative error of $\varepsilon_{\boldsymbol{A}}^{(2 K)}=5 \%$ so that we think that the system behaves as $\hat{\boldsymbol{A}}=\boldsymbol{A}+\boldsymbol{E}$. From (8) we can verify that matrix $\hat{\boldsymbol{A}}$ has an RIC $\hat{\delta}_{2 K, \max }=0.213$ which satisfies (12). Thus, if (13) is also satisfied, then Theorem 2 guarantees that the BP solution will have accuracy given in (15) with $C_{0}=4.47$ and $C_{1}=9.06$. Note from (16) and (17) we see that if there had been no perturbation, then $C_{0}=2.75$ and $C_{1}=5.53$.

Consider now a different example. Suppose instead that $\delta_{2 K}=0.200$ with $\varepsilon_{\boldsymbol{A}}^{(2 K)}=1 \%$. Then $\hat{\delta}_{2 K \text {, max }}=0.224$, $C_{0}=4.76$ and $C_{1}=9.64$. Here, if $\boldsymbol{A}$ was unperturbed, then we would have had $C_{0}=4.19$ and $C_{1}=8.47$.

These numerical examples show how the stability constants $C_{0}$ and $C_{1}$ of the BP solution get worse with perturbations to $\boldsymbol{A}$. It must be stressed however, that they represent worst-case instances. It is well-known in the CS community that better performance is normally achieved in practice.

\section{Numerical Simulations}

Numerical simulations were conducted in MATLAB as follows. In each trial a new matrix $\boldsymbol{A}$ of size $128 \times 512$ was randomly generated with normally distributed entries $\mathcal{N}\left(0, \sigma^{2}\right)$ where $\sigma^{2}=1 / 128$ (so that the expected $\ell_{2}$ norm of each column was unity), and the spectral norm of $\boldsymbol{A}$ was calculated. Next, for each relative perturbation $\varepsilon_{\boldsymbol{A}}=0,0.01,0.05,0.1$ a different perturbation matrix $\boldsymbol{E}$ with normally distributed entries was generated, and then scaled so that $\|\boldsymbol{E}\|_{2}=\varepsilon_{\boldsymbol{A}} \cdot\|\boldsymbol{A}\|_{2}, 7$ A random vector $\boldsymbol{x}$ of sparsity $K=1, \ldots, 64$ was then randomly generated with nonzero entries uniformly distributed $\mathcal{N}(0,1)$, and $\hat{\boldsymbol{b}}=\boldsymbol{A} \boldsymbol{x}$ in (2) was created (note, we set $\boldsymbol{e}=\mathbf{0}$ so as to focus on the effect of perturbation $\boldsymbol{E}$ ). Finally, given $\hat{\boldsymbol{b}}$ and the $\hat{\boldsymbol{A}}=\boldsymbol{A}+\boldsymbol{E}$ associated with each $\varepsilon_{\boldsymbol{A}}$, the BP program (7) was implemented with Cvx software [11] and the relative error $\left\|\boldsymbol{z}^{\star}-\boldsymbol{x}\right\|_{2} /\|\boldsymbol{x}\|_{2}$ was recorded. One hundred trials were performed for each value of $K$.

Figure 1 shows the relative error averaged over the 100 trials as a function of $K$ for each $\varepsilon_{A}$. As a reference, the ideal, noise-free case can be seen for $\varepsilon_{\boldsymbol{A}}=0$. Now fix a particular value of $K \leq 30$ and compare the relative error for the three nonzero values of $\varepsilon_{\boldsymbol{A}}$. It is clear that the error scales roughly linearly with $\varepsilon_{\boldsymbol{A}}$. For example, when $K=10$ the relative errors corresponding to $\varepsilon_{\boldsymbol{A}}=0.01,0.05,0.1$ respectively are $9.7 \times 10^{-3}, 4.9 \times 10^{-2}, 9.7 \times 10^{-2}$. We see here that the relative errors for $\varepsilon_{\boldsymbol{A}}=0.05$ and 0.1 are approximately five and ten times the relative error associated with $\varepsilon_{\boldsymbol{A}}=0.01$. Therefore, this empirical study essentially confirms the conclusion of Theorem 2 the stability of the BP solution scales linearly with $\varepsilon_{\boldsymbol{A}}^{(K)}$.

Note that improved performance in theory and in simulation can be achieved if BP is used solely to determine the support of the solution. Then we can use least squares to better approximate the coefficients on this support. This is similar to the the best-case, oracle least squares solution discussed in Section IV However, this method of recovery was not pursued in the present analysis.

\section{PROOFS}

\section{A. Proof of Theorem 1}

Recall that we are tasked with determining the maximum $\hat{\delta}_{K}$ given $\delta_{K}$ and $\varepsilon_{\boldsymbol{A}}^{(K)}$. Temporarily define $l_{K}$ and $u_{K}$ as the smallest nonnegative numbers such that

$$
\left(1-l_{K}\right)\|\boldsymbol{x}\|_{2}^{2} \leq\|\hat{\boldsymbol{A}} \boldsymbol{x}\|_{2}^{2} \leq\left(1+u_{K}\right)\|\boldsymbol{x}\|_{2}^{2}
$$

\footnotetext{
${ }^{7}$ We used $\varepsilon_{\boldsymbol{A}}$ in these simulations since calculating $\varepsilon_{\boldsymbol{A}}^{(K)}$ explicitly is extremely difficult. Notice that $\varepsilon_{\boldsymbol{A}} \approx \varepsilon_{\boldsymbol{A}}^{(K)}$ for all $K$ with high probability since both $\boldsymbol{A}, \boldsymbol{E}$ are random Gaussian matrices.
} 


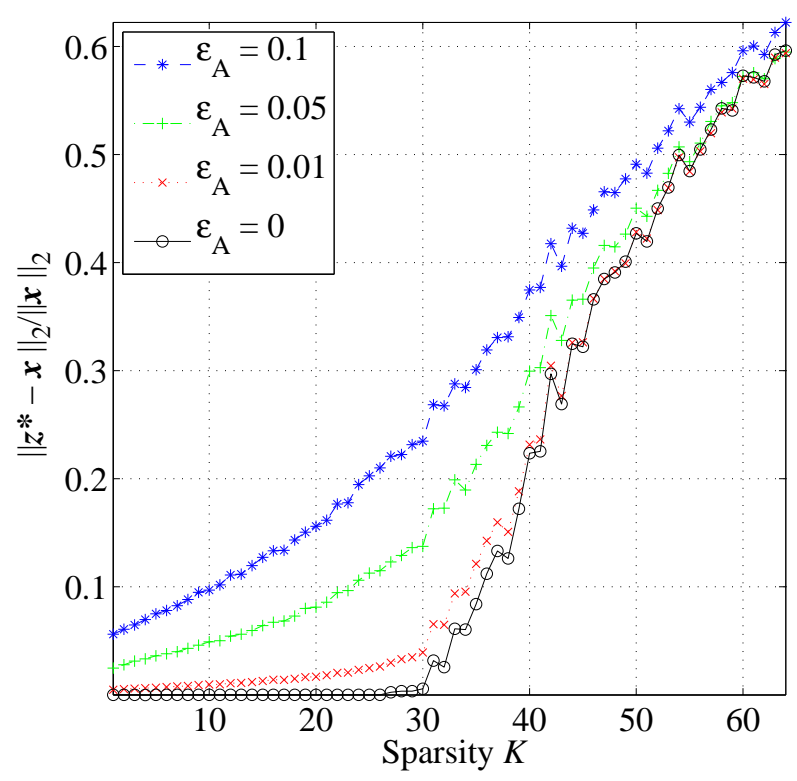

Fig. 1. Average (100 trials) relative error of BP solution $\boldsymbol{z}^{\star}$ with respect to $K$-sparse $\boldsymbol{x}$ vs. Sparsity $K$ for different relative perturbations $\varepsilon_{\boldsymbol{A}}$ of $\boldsymbol{A}$. Here $\boldsymbol{A}, \boldsymbol{E}$ are both $128 \times 512$ random matrices with i.i.d. Gaussian entries and $\varepsilon_{\boldsymbol{b}}=0$.

holds for any $K$-sparse vector $\boldsymbol{x}$. From the triangle inequality, (5) and (3) we have

$$
\begin{aligned}
\|\hat{\boldsymbol{A}} \boldsymbol{x}\|_{2}^{2} & \leq\left(\|\boldsymbol{A} \boldsymbol{x}\|_{2}+\|\boldsymbol{E} \boldsymbol{x}\|_{2}\right)^{2} \\
& \leq\left(\sqrt{1+\delta_{K}}+\|\boldsymbol{E}\|_{2}^{(K)}\right)^{2}\|\boldsymbol{x}\|_{2}^{2} \\
& \leq\left(1+\delta_{K}\right)\left(1+\varepsilon_{\boldsymbol{A}}^{(K)}\right)^{2}\|\boldsymbol{x}\|_{2}^{2} .
\end{aligned}
$$

In comparing the RHS of (19) and (22), it must be that

$$
\left(1+u_{K}\right) \leq\left(1+\delta_{K}\right)\left(1+\varepsilon_{\boldsymbol{A}}^{(K)}\right)^{2}
$$

as demanded by the definition of the $u_{K}$. Moreover, this inequality is sharp for the following reasons:

- Equality occurs in (20) whenever $\boldsymbol{E}$ is a positive, real-valued multiple of $\boldsymbol{A}$.

- The inequality in (21) inherits the sharpness of the upper bound of the RIP for matrix $\boldsymbol{A}$ in (5).

- Equality occurs in (22) since, in this hypothetical case, we assume that $\boldsymbol{E}=\beta \boldsymbol{A}$ for some $0<\beta<1$. Therefore, the relative perturbation $\varepsilon_{\boldsymbol{A}}^{(K)}$ in (3) no longer represents a worst-case deviation (i.e., the ratio $\left.\frac{\|\boldsymbol{E}\|_{2}^{(K)}}{\|\boldsymbol{A}\|_{2}^{(K)}}=\beta=: \varepsilon_{\boldsymbol{A}}^{(K)}\right)$.

Since the triangle inequality constitutes a least-upper bound, and since we attain this bound, then

$$
u_{K}:=\left(1+\delta_{K}\right)\left(1+\varepsilon_{\boldsymbol{A}}^{(K)}\right)^{2}-1
$$

satisfies the definition of $u_{K}$.

Now the LHS of (19) is obtained in much the same way using the "reverse" triangle inequality with similar arguments (in particular, assume $-1<\beta<0$ and $\varepsilon_{\boldsymbol{A}}^{(K)}:=|\beta|$ ). Thus

$$
l_{K}:=1-\left(1-\delta_{K}\right)\left(1-\varepsilon_{\boldsymbol{A}}^{(K)}\right)^{2} .
$$

Next, we need to make the bounds of (19) symmetric. Notice that $\left(1-u_{K}\right) \leq\left(1-l_{K}\right)$ and $\left(1+l_{K}\right) \leq\left(1+u_{K}\right)$. Therefore, given $\delta_{K}$ and $\varepsilon_{\boldsymbol{A}}^{(K)}$, we choose

$$
\hat{\delta}_{K, \max }:=u_{K}
$$

as the smallest nonnegative constant which makes (19) symmetric. Finally, it is clear that the actual RIC $\hat{\delta}_{K}$ for $\hat{A}$ obeys $\hat{\delta}_{K} \leq \hat{\delta}_{K \text {, max }}$. Hence, (9) follows immediately. 


\section{B. Bounding the perturbed observation}

Before proceeding to the proof of Theorem 2 we need several important facts. First we generalize a lemma in [12] about the image of an arbitrary signal.

Proposition 1 ([12], Lemma 29). Assume that matrix $A$ satisfies the upper bound of the RIP in (5). Then for every signal $\boldsymbol{x}$ we have

$$
\|\boldsymbol{A} \boldsymbol{x}\|_{2} \leq \sqrt{1+\delta_{K}}\left(\|\boldsymbol{x}\|_{2}+\frac{1}{\sqrt{K}}\|\boldsymbol{x}\|_{1}\right) .
$$

Now we can establish sufficient conditions for the lower bound in terms of the head and tail of $\boldsymbol{x}$ and the RIC of $\boldsymbol{A}$.

Lemma 2. Assume condition (13) in Theorem 2 Then for general signal $\boldsymbol{x}$, its image under $\boldsymbol{A}$ can be bounded below by the positive quantity

$$
\|\boldsymbol{A} \boldsymbol{x}\|_{2} \geq \sqrt{1-\delta_{K}}\left(\left\|\boldsymbol{x}_{K}\right\|_{2}-\kappa_{\boldsymbol{A}}^{(K)}\left(\left\|\boldsymbol{x}_{K^{c}}\right\|_{2}+\frac{\left\|\boldsymbol{x}_{K^{c}}\right\|_{1}}{\sqrt{K}}\right)\right) .
$$

Proof: Apply Proposition 1 to the tail of $\boldsymbol{x}$. Then

$$
\begin{aligned}
\|\boldsymbol{A} \boldsymbol{x}\|_{2} & \geq\left\|\boldsymbol{A} \boldsymbol{x}_{K}\right\|_{2}-\left\|\boldsymbol{A} \boldsymbol{x}_{K^{c}}\right\|_{2} \\
& \geq \sqrt{1-\delta_{K}}\left\|\boldsymbol{x}_{K}\right\|_{2}-\sqrt{1+\delta_{K}}\left(\left\|\boldsymbol{x}_{K^{c}}\right\|_{2}+\frac{\left\|\boldsymbol{x}_{K^{c}}\right\|_{1}}{\sqrt{K}}\right) \\
& =\sqrt{1-\delta_{K}}\left(1-\kappa_{\boldsymbol{A}}^{(K)}\left(r_{K}+\frac{s_{K}}{\sqrt{K}}\right)\right)\left\|\boldsymbol{x}_{K}\right\|_{2} \\
& >0
\end{aligned}
$$

on account of (13).

We still need some sense of the size of the total perturbation incurred by $\boldsymbol{E}$ and $e$. We do not know a priori the exact values of $\boldsymbol{E}, \boldsymbol{x}$, or $\boldsymbol{e}$. But we can find an upper bound in terms of the relative perturbations in (3). The main goal in the following lemma is to remove the total perturbation's dependence on the input $\boldsymbol{x}$.

Lemma 3 (Total perturbation bound). Assume condition (13) in Theorem 2 and set 8

$$
\varepsilon_{\boldsymbol{A}, K, \boldsymbol{b}}^{\prime}:=\left(\frac{\varepsilon_{\boldsymbol{A}}^{(K)} \kappa_{\boldsymbol{A}}^{(K)}+\varepsilon_{\boldsymbol{A}} \alpha_{\boldsymbol{A}} r_{K}}{1-\kappa_{\boldsymbol{A}}^{(K)}\left(r_{K}+s_{K} / \sqrt{K}\right)}+\varepsilon_{\boldsymbol{b}}\right)\|\boldsymbol{b}\|_{2}
$$

where $\varepsilon_{\boldsymbol{A}}, \varepsilon_{\boldsymbol{A}}^{(K)}, \varepsilon_{\boldsymbol{b}}$ are defined in (3), $\kappa_{\boldsymbol{A}}^{(K)}, \alpha_{\boldsymbol{A}}$ in (10), and $r_{K}, s_{K}$ in (11). Then the total perturbation obeys

$$
\|\boldsymbol{E} \boldsymbol{x}\|_{2}+\|\boldsymbol{e}\|_{2} \leq \varepsilon_{\boldsymbol{A}, K, \boldsymbol{b}}^{\prime}
$$

Proof: First divide the multiplicative noise term by $\|\boldsymbol{b}\|_{2}$ and then apply Lemma 2

$$
\begin{aligned}
\frac{\|\boldsymbol{E} \boldsymbol{x}\|_{2}}{\|\boldsymbol{A} \boldsymbol{x}\|_{2}} & \leq \frac{\left(\|\boldsymbol{E}\|_{2}^{(K)}\left\|\boldsymbol{x}_{K}\right\|_{2}+\|\boldsymbol{E}\|_{2}\left\|\boldsymbol{x}_{K^{c}}\right\|_{2}\right) \cdot \frac{1}{\sqrt{1-\delta_{K}}}}{\left\|\boldsymbol{x}_{K}\right\|_{2}-\kappa_{\boldsymbol{A}}^{(K)}\left(\left\|\boldsymbol{x}_{K^{c}}\right\|_{2}+\left\|\boldsymbol{x}_{K^{c}}\right\|_{1} / \sqrt{K}\right)} \\
& =\frac{\left(\|\boldsymbol{E}\|_{2}^{(K)}+\|\boldsymbol{E}\|_{2} r_{K}\right) \cdot \frac{1}{\sqrt{1-\delta_{K}}}}{1-\kappa_{\boldsymbol{A}}^{(K)}\left(r_{K}+s_{K} / \sqrt{K}\right)} \\
& \leq \frac{\varepsilon_{\boldsymbol{A}}^{(K)} \kappa_{\boldsymbol{A}}^{(K)}+\varepsilon_{\boldsymbol{A}} \alpha_{\boldsymbol{A}} r_{K}}{1-\kappa_{\boldsymbol{A}}^{(K)}\left(r_{K}+s_{K} / \sqrt{K}\right)} .
\end{aligned}
$$

Including the contribution from the additive noise term completes the proof.

\footnotetext{
${ }^{8}$ Note that the results in this paper can easily be expressed in terms of the perturbed observation by replacing $\|\boldsymbol{b}\|_{2} \leq\|\hat{\boldsymbol{b}}\|_{2}\left(1-\varepsilon_{\boldsymbol{b}}\right)^{-1}$. This can be useful in practice since one normally only has access to $\hat{b}$.
} 


\section{Proof of Theorem 2}

Step 1. We duplicate the techniques used in Candès' proof of Theorem 1.2 in [1], but with decoding matrix $\boldsymbol{A}$ replaced by $\hat{\boldsymbol{A}}$. The proof relies heavily on the RIP for $\hat{\boldsymbol{A}}$ in Theorem 1 Set the BP minimizer in (7) as $\boldsymbol{z}^{\star}=\boldsymbol{x}+\boldsymbol{h}$. Here, $\boldsymbol{h}$ is the perturbation from the true solution $\boldsymbol{x}$ induced by $\boldsymbol{E}$ and $\boldsymbol{e}$. Instead of Candès' (9), we now determine that the image of $\boldsymbol{h}$ under $\hat{A}$ is bounded by

$$
\begin{aligned}
\|\hat{\boldsymbol{A}} \boldsymbol{h}\|_{2} & \leq\left\|\hat{\boldsymbol{A}} \boldsymbol{z}^{\star}-\hat{\boldsymbol{b}}\right\|_{2}+\|\hat{\boldsymbol{A}} \boldsymbol{x}-\hat{\boldsymbol{b}}\|_{2} \\
& \leq 2 \varepsilon_{\boldsymbol{A}, K, \boldsymbol{b}}^{\prime} .
\end{aligned}
$$

The second inequality follows since both terms on the RHS of (25) satisfy the BP constraint in (7). Notice in the second term that $\boldsymbol{x}$ is a feasible solution due to Lemma 3 .

Since the other steps in the proof are essentially the same, we end up with constants $\hat{\alpha}$ and $\hat{\rho}$ in Candès' (14) (instead of $\alpha$ and $\rho$ ) where

$$
\hat{\alpha}:=\frac{2 \sqrt{1+\hat{\delta}_{2 K}}}{1-\hat{\delta}_{2 K}}, \quad \hat{\rho}:=\frac{\sqrt{2} \hat{\delta}_{2 K}}{1-\hat{\delta}_{2 K}} .
$$

The final line of the proof concludes that

$$
\|\boldsymbol{h}\|_{2} \leq \frac{2 \hat{\alpha}(1+\hat{\rho})}{1-\hat{\rho}} \frac{\left\|\boldsymbol{x}-\boldsymbol{x}_{K}\right\|_{1}}{\sqrt{K}}+\frac{2 \hat{\alpha}}{1-\hat{\rho}} \varepsilon_{\boldsymbol{A}, K, \boldsymbol{b}}^{\prime}
$$

The denominator demands that we impose the condition that $0<1-\hat{\rho}$, or equivalently

$$
\hat{\delta}_{2 K}<\sqrt{2}-1 \text {. }
$$

The constants $C_{0}$ and $C_{1}$ are obtained by first substituting $\hat{\alpha}$ and $\hat{\rho}$ from 26 into 27). Then, recalling that $\hat{\delta}_{2 K} \leq \hat{\delta}_{2 K \text {, max }}$, substitute $\hat{\delta}_{K \text {, max }}$ from (18) (with $K \rightarrow 2 K$ ).

Step 2. We still need to show that the hypothesis of Theorem 2 implies (28). This is easily verified by substituting the assumption of $\delta_{2 K}<\sqrt{2}\left(1+\varepsilon_{\boldsymbol{A}}^{(2 K)}\right)^{-2}-1$ into (8) (again with $K \rightarrow 2 K$ ) and the proof is complete.

\section{Proof of Lemma 1}

Assume (12) in the hypothesis of Theorem 2, It is easy to show that this implies

$$
\|\boldsymbol{E}\|_{2}^{(2 K)}<\sqrt[4]{2}-\sqrt{1+\delta_{2 K}} .
$$

Simple algebraic manipulation then confirms that

$$
\sqrt[4]{2}-\sqrt{1+\delta_{2 K}}<\sqrt{1-\delta_{2 K}} \leq \sigma_{\min }^{(2 K)}(\boldsymbol{A}) .
$$

Therefore, (18) holds with $k=2 K$. Further, for any $k \leq 2 K$ we have $\sigma_{\max }^{(k)}(\boldsymbol{E}) \leq \sigma_{\max }^{(2 K)}(\boldsymbol{E})$ and $\sigma_{\min }^{(2 K)}(\boldsymbol{A}) \leq$ $\sigma_{\min }^{(k)}(\boldsymbol{A})$, which proves the first part of the lemma. The second part is an immediate consequence.

\section{Classical $\ell_{2}$ Perturbation Analysis}

Let the subset $T \subseteq\{1, \ldots, n\}$ have cardinality $|T|=K$, and note the following T-restrictions: $\boldsymbol{A}_{T} \in \mathbb{C}^{m \times K}$ denotes the submatrix consisting of the columns of $\boldsymbol{A}$ indexed by the elements of $T$, and similarly for $\boldsymbol{x}_{T} \in \mathbb{C}^{K}$.

Suppose the "oracle" case where we already know the support $T$ of $\boldsymbol{x}_{K}$, i.e., the best $K$-sparse representation of $\boldsymbol{x} 9$ By assumption, we are only interested in the case where $K \leq m$ in which $\boldsymbol{A}_{T}$ has full rank. Given the completely perturbed observation of (2), the least squares problem consists of solving:

$$
\boldsymbol{z}_{T}^{\#}=\underset{\hat{\boldsymbol{z}}_{T}}{\operatorname{argmin}}\left\|\hat{\boldsymbol{A}}_{T} \hat{\boldsymbol{z}}_{T}-\hat{\boldsymbol{b}}\right\|_{2}
$$

\footnotetext{
${ }^{9}$ Although perhaps slightly confusing, note that $\boldsymbol{x}_{K} \in \mathbb{C}^{n}$, while $\boldsymbol{x}_{T} \in \mathbb{C}^{K}$. Restricting $\boldsymbol{x}_{K}$ to its support $T$ yields $\boldsymbol{x}_{T}$.
} 
Since we know the support $T$, it is trivial to extend $\boldsymbol{z}_{T}^{\#}$ to $\boldsymbol{z}^{\#} \in \mathbb{C}^{n}$ by zero-padding on the complement of $T$. Our goal is to see how the perturbations $\boldsymbol{E}$ and $\boldsymbol{e}$ affect $\boldsymbol{z}^{\#}$. Using Golub and Van Loan's model ([13], Thm. 5.3.1) as a guide, assume

$$
\max \left\{\frac{\left\|\boldsymbol{E}_{T}\right\|_{2}}{\left\|\boldsymbol{A}_{T}\right\|_{2}}, \frac{\|\boldsymbol{e}\|_{2}}{\|\boldsymbol{b}\|_{2}}\right\}<\frac{\sigma_{\min }\left(\boldsymbol{A}_{T}\right)}{\sigma_{\max }\left(\boldsymbol{A}_{T}\right)} .
$$

Remark 4. This assumption is fairly easy to satisfy. In fact, assumption (12) in the hypothesis of Theorem 2 immediately implies that $\left\|\boldsymbol{E}_{T}\right\|_{2} /\left\|\boldsymbol{A}_{T}\right\|_{2}<\sigma_{\min }\left(\boldsymbol{A}_{T}\right) / \sigma_{\max }\left(\boldsymbol{A}_{T}\right)$ for all $\varepsilon_{\boldsymbol{A}}^{(2 K)} \in[0, \sqrt[4]{2}-1)$. To see this simply set $k=K$ in 18 of Lemma 1 , and note that $\left\|\boldsymbol{E}_{T}\right\|_{2} \leq\|\boldsymbol{E}\|_{2}^{(K)}$ and $\sigma_{\min }^{(K)}(\boldsymbol{A}) \leq \sigma_{\min }\left(\boldsymbol{A}_{T}\right)$. Further, the reasonable condition of $\varepsilon_{\boldsymbol{b}} \leq\left(\sqrt{2}\left(1+\varepsilon_{\boldsymbol{A}}^{(2 K)}\right)^{2}-1\right)^{1 / 2}$ is sufficient to ensure $\varepsilon_{\boldsymbol{b}}<\sqrt{1-\delta_{2 K}} / \sqrt{1+\delta_{2 K}}$ so that assumption (29) holds. Note that this assumption has no bearing on CS recovery, nor is it a constraint due to BP. It is simply made to enable an analysis of the least squares solution which we use as a best-case comparison below.

Following the steps in [13] with the appropriate modifications for our situation we obtain

$$
\begin{aligned}
\left\|\boldsymbol{z}^{\#}-\boldsymbol{x}_{K}\right\|_{2} & \leq\left\|\boldsymbol{A}_{T}^{\dagger}\right\|_{2}\left(\frac{\left\|\boldsymbol{E}_{T} \boldsymbol{x}_{T}\right\|_{2}}{\|\boldsymbol{A} \boldsymbol{x}\|_{2}}+\frac{\|\boldsymbol{e}\|_{2}}{\|\boldsymbol{b}\|_{2}}\right)\|\boldsymbol{b}\|_{2} \\
& \leq \frac{1}{\sqrt{1-\delta_{K}}} \zeta_{\boldsymbol{A}, K, \boldsymbol{b}}^{\prime}
\end{aligned}
$$

where $\boldsymbol{A}_{T}^{\dagger}=\left(\boldsymbol{A}_{T}^{*} \boldsymbol{A}_{T}\right)^{-1} \boldsymbol{A}_{T}^{*}$ is the left inverse of $\boldsymbol{A}_{T}$ whose spectral norm

$$
\left\|\boldsymbol{A}_{T}^{\dagger}\right\|_{2} \leq \frac{1}{\sqrt{1-\delta_{K}}}
$$

and where

$$
\zeta_{\boldsymbol{A}, K, \boldsymbol{b}}^{\prime}:=\left(\frac{\kappa_{\boldsymbol{A}}^{(K)} \varepsilon_{\boldsymbol{A}}^{(K)}}{1-\kappa_{\boldsymbol{A}}^{(K)}\left(r_{K}+s_{K} / \sqrt{K}\right)}\right)\|\boldsymbol{b}\|_{2}
$$

was obtained using the same steps as in (24). Finally, we obtain the total least squares stability expression

$$
\begin{aligned}
\left\|\boldsymbol{z}^{\#}-\boldsymbol{x}\right\|_{2} & \leq\left\|\boldsymbol{x}-\boldsymbol{x}_{K}\right\|_{2}+\left\|\boldsymbol{z}^{\#}-\boldsymbol{x}_{K}\right\|_{2} \\
& \leq\left\|\boldsymbol{x}-\boldsymbol{x}_{K}\right\|_{2}+C_{2} \zeta_{\boldsymbol{A}, K, \boldsymbol{b}}^{\prime}
\end{aligned}
$$

with $C_{2}=1 / \sqrt{1-\delta_{K}}$.

\section{A. Comparison of $L S$ with BP}

Now, we can compare the accuracy of the least squares solution in (30) with the accuracy of the BP solution found in (15). However, this comparison is not really appropriate when the original data is compressible since the least squares solution $z^{\#}$ returns a vector which is strictly $K$-sparse, while the BP solution $z^{\star}$ will never be strictly sparse.

To make the comparison fair, we need to assume that $\boldsymbol{x}$ is strictly $K$-sparse. Then, as mentioned previously, the constants $r_{K}=s_{K}=0$ and the solutions enjoy stability of

$$
\left\|\boldsymbol{z}^{\#}-\boldsymbol{x}\right\|_{2} \leq C_{2}\left(\kappa_{\boldsymbol{A}}^{(K)} \varepsilon_{\boldsymbol{A}}^{(K)}+\varepsilon_{\boldsymbol{b}}\right)\|\boldsymbol{b}\|_{2}
$$

and

$$
\left\|\boldsymbol{z}^{\star}-\boldsymbol{x}\right\|_{2} \leq C_{1}\left(\kappa_{\boldsymbol{A}}^{(K)} \varepsilon_{\boldsymbol{A}}^{(K)}+\varepsilon_{\boldsymbol{b}}\right)\|\boldsymbol{b}\|_{2} .
$$

Yet, a detailed numerical comparison of $C_{2}$ with $C_{1}$, even at this point, is still is not entirely valid, nor illuminating. This is due to the fact that we assumed the oracle setup in the least squares analysis, which is the best that one could hope for. In this sense, the least squares solution we examined here can be considered a "best, worst-case" scenario. In contrast, the BP solution really should be thought of as a "worst, of the worst-case" scenarios.

The important thing to glean is that the accuracy of the BP and the least squares solutions are both on the order of the noise level

$$
\left(\kappa_{\boldsymbol{A}}^{(K)} \varepsilon_{\boldsymbol{A}}^{(K)}+\varepsilon_{\boldsymbol{b}}\right)\|\boldsymbol{b}\|_{2}
$$

in the perturbed observation. This is an important finding since, in general, no other recovery algorithm can do better than the oracle least squares solution. These results are analogous to the comparison by Candès, Romberg and Tao in [2], although they only consider the case of additive noise $\boldsymbol{e}$. 


\section{CONCLUSiON}

We introduced a framework to analyze general perturbations in CS and found the conditions under which BP could stably recover the original data. This completely perturbed model extends previous work by including a multiplicative noise term in addition to the usual additive noise term.

Most of this study assumed no specific knowledge of the perturbations $\boldsymbol{E}$ and $\boldsymbol{e}$. Instead, the point of view was in terms of their worst-case relative perturbations $\varepsilon_{\boldsymbol{A}}, \varepsilon_{\boldsymbol{A}}^{(K)}, \varepsilon_{\boldsymbol{b}}$. In real-world applications these quantities must either be calculated or estimated. This must be done with care owing to their role in the theorems presented here.

We derived the RIP for perturbed matrix $\hat{\boldsymbol{A}}$, and showed that the penalty on the spectrum of its $K$-column submatrices was a graceful, linear function of the relative perturbation $\varepsilon_{\boldsymbol{A}}^{(K)}$. Our main contribution, Theorem 2 showed that the stability of the BP solution of the complectly perturbed scenario was limited by the total noise in the observation.

Simple numerical examples demonstrated how the multiplicative noise reduced the accuracy of the recovered BP solution. Formal numerical simulations were performed on strictly $K$-sparse signals with no additive noise so as to highlight the effect of perturbation $\boldsymbol{E}$. These experiments appear to confirm the conclusion of Theorem 2 , the stability of the BP solution scales linearly with $\varepsilon_{\boldsymbol{A}}^{(K)}$.

We also found that the rank of $\hat{\boldsymbol{A}}$ did not exceed the rank of $\boldsymbol{A}$ under the assumed conditions. This permitted a comparison with the oracle least squares solution.

It should be mentioned that designing matrices and checking for proper RICs is still quite elusive. In fact, the only matrices which are known to satisfy the RIP (and which have $m \sim K$ rows) are random Gaussian, Bernoulli, and certain partial unitary (e.g., Fourier) matrices (see, e.g., [14], [15], [16]).

\section{APPENDIX}

\section{DIFFERENT CASES OF PERTURBATION $\boldsymbol{E}$}

There are essentially two classes of perturbations $\boldsymbol{E}$ which we care most about: random and structured. The nature of these perturbation matrices will have a significant effect on the value of $\|\boldsymbol{E}\|_{2}^{(K)}$, which is used in determining $\varepsilon_{\boldsymbol{A}}^{(K)}$ in (3). In fact, explicit knowledge of $\boldsymbol{E}$ can significantly improve the worst-case assumptions presented throughout this paper. However, if there is no extra knowledge on the nature of $\boldsymbol{E}$, then we can rely on the "worst case" upper bound using the full matrix spectral norm: $\|\boldsymbol{E}\|_{2}^{(K)} \leq\|\boldsymbol{E}\|_{2}$.

\section{A. Random Perturbations}

Random matrices, such as Gaussian, Bernoulli, and certain partial Fourier matrices, are often amenable to analysis with the RIP. For instance, suppose that $\boldsymbol{E}$ is simply a scaled version of a random matrix $\boldsymbol{R}$ so that $\boldsymbol{E}=\beta \boldsymbol{R}$ with $0<\beta \ll 1$. Denote $\delta_{K}^{\boldsymbol{R}}$ as the RIC associated with the matrix $\boldsymbol{R}$. Then for all $K$-sparse $\boldsymbol{x}$ the RIP for matrix $\boldsymbol{E}$ asserts

$$
\beta^{2}\left(1-\delta_{K}^{\boldsymbol{R}}\right)\|\boldsymbol{x}\|_{2}^{2} \leq\|\boldsymbol{E} \boldsymbol{x}\|_{2}^{2} \leq \beta^{2}\left(1+\delta_{K}^{\boldsymbol{R}}\right)\|\boldsymbol{x}\|_{2}^{2},
$$

which immediately gives us

$$
\|\boldsymbol{E}\|_{2}^{(K)} \leq \beta \sqrt{1+\delta_{K}^{R}}
$$

and thus

$$
\frac{\|\boldsymbol{E}\|_{2}^{(K)}}{\|\boldsymbol{A}\|_{2}^{(K)}} \leq \beta \frac{\sqrt{1+\delta_{K}^{\boldsymbol{R}}}}{\sqrt{1-\delta_{K}}}=: \varepsilon_{\boldsymbol{A}}^{(K)}
$$

\section{B. Structured Perturbations}

Structured matrices (e.g., Toeplitz, banded) are ubiquitous in the mathematical sciences and engineering. In the CS scenario, suppose for example that $\boldsymbol{E}$ is a partial circulant matrix obtained by selecting $m$ rows uniformly at random from an $n \times n$ circulant matrix. An error in the modeling of a communication channel could be represented by such a partial circulant matrix. When encountering a structured perturbation such as this it may be possible to exploit its nature to find a bound $\|\boldsymbol{E}\|_{2}^{(K)} \leq C$. 
A complete circulant matrix has the property that each row is simply a right-shifted version of the row above it. Therefore, knowledge of any row gives information about the entries of all of the rows. This is also true for a partial circulant matrix. Thus, with this information we may be able to find a reasonable upper bound on $\|\boldsymbol{E}\|_{2}^{(K)}$. The interested reader can find relevant literature at [17].

\section{ACKNOWLEDGMENT}

The authors would like to thank Jeffrey Blanchard at the University of Utah, Deanna Needell and Albert Fannjiang at the University of California, Davis and the anonymous reviewers. Their comments and suggestions helped to make the current version of this paper much stronger.

\section{REFERENCES}

[1] E. J. Candès, “The restricted isometry property and its implications for compressed sensing," Académie des Sciences, vol. I, no. 346, pp. 589-592, 2008.

[2] E. J. Candès, J. Romberg, and T. Tao, "Stable signal recovery from incomplete and inaccurate measurements," Comm. Pure Appl. Math., vol. 59, pp. 1207-1223, 2006.

[3] D. L. Donoho, M. Elad, and V. Temlyakov, "Stable recovery of sparse overcomplete representations in the presence of noise," IEEE Trans. Inf. Theory, vol. 52, no. 1, pp. 6-18, Jan. 2006.

[4] J. A. Tropp, "Just relax: Convex programming methods for identifying sparse signals in noise," IEEE Trans. Inf. Theory, vol. 51, no. 3, pp. 1030-1051, Mar. 2006.

[5] R. Gribonval, H. Rauhut, K. Schnass, and P. Vandergheynst, "Atoms of all channels, unite! Average case analysis of multi-channel sparse recovery using greedy algorithms," Journal of Fourier Analysis and Applications, vol. 14, no. 5-6, pp. 1069-5869, Dec. 2008.

[6] T. Blumensath and M. Davies, "Compressed sensing and source separation," Inter. Conf. on Ind. Comp. Anal. and Source Sep., pp. 341-348, Sept. 2007.

[7] M. A. Herman and T. Strohmer, "High-resolution radar via compressed sensing," IEEE Trans. Sig. Proc., vol. 57, no. 6, pp. 2275-2284, Jun. 2009

[8] A. Fannjiang, P. Yan, and T. Strohmer, "Compressed remote sensing of sparse objects," submitted April 2009.

[9] S. S. Chen, D. L. Donoho, and M. A. Saunders, "Atomic decomposition by basis pursuit," SIAM Journal Sci. Comput., vol. 20, no. 1, pp. 33-61, 1999.

[10] E. J. Candès and T. Tao, "Decoding by linear programming," IEEE Trans. Inf. Theory, vol. 51, no. 12, pp. 4203-4215, Dec. 2005.

[11] M. Grant, S. Boyd, and Y. Ye, "cvx: Matlab software for disciplined convex programming," http://www.stanford.edu/ boyd/cvx/.

[12] A. C. Gilbert, M. J. Strauss, J. A. Tropp, and R. Vershynin, "One sketch for all: Fast algorithms for compressed sensing," Proc. 39th ACM Symp. Theory of Computing (STOC), San Diego, CA, Jun. 2007.

[13] G. H. Golub and C. F. Van Loan, Matrix Computations, 3rd ed. Baltimore: Johns Hopkins University Press, 1996.

[14] E. J. Candès and T. Tao, "Near-optimal signal recovery from random projections: Universal encoding strategies," IEEE Trans. Inf. Theory, vol. 52, pp. 5406-5425, 2006.

[15] S. Mendelson, A. Pajor, and N. Tomczak-Jaegermann, "Uniform uncertainty principle for Bernoulli and subgaussian ensembles," 2009, to appear, Constr. Approx.

[16] M. Rudelson and R. Vershynin, "On sparse reconstruction from Fourier and Gaussian measurements," Communications on Pure and Applied Mathematics, vol. 61, pp. 1025-1045, 2008.

[17] The Rice University Compressive Sensing Resources web page, http://www.dsp.ece.rice.edu/cs. 